UNITED STATES DEPARTMENT OF THE INTERIOR

GEOLOGICAL: SURVEY

\title{
Prediction of Vertical Displacements in a Subsiding Elastic Layer--A Model for \\ Subsidence in Karst Terrains
}

\section{By}

William Z. Savage

Open-File Report 79-1094

1979

This report is preliminary and has not been edited or reviewed for conformity with U.S. Geological Survey standards and nomenclature. 


\section{CONTENTS}

Page

Introduction.............................................. I

The two boundary-value problems............................. 2

Evaluation of vertical displacements on the free surface............ 6

Vertical displacements on the free surface and over the opening...... 8

Discussion of resulting displacements.......................... 11

References.............................................. 12 


\section{ILLUSTRATIONS}

Page

Figure 1. Elastic layer of thickness $h$ overlying an opening of width 2 a..................................... 3

2. Vertical displacements on the free surface as a function of $n=x / a$ and $\delta=h / a \ldots \ldots \ldots \ldots \ldots \ldots . .9$

3. Vertical displacements above the hole (at $y=0) \ldots \ldots \ldots .10$ 
Notation
a
half-width of opening
h
layer thickness
$\varepsilon_{i j}$
strains
$\sigma_{\mathbf{i j}}$
stresses
v
vertical displacement
v
Poisson's ratio
E
Young's modulus
p
density
g
acceleration due to gravity
$x, y$
horizontal and vertical coordinates
$\delta=h / a$
ratio of layer thickness to half-width of opening
$\alpha$
constant
$m_{1}, m_{3}$
constants
$n=x / a$
ratio of $x$ coordinate to half-width of opening
$v_{0}, v_{g}$
constants
$u_{n}$
Bessel function of order $n$ 
Prediction of vertical displacements in a subsiding elastic layer--A model for subsidence in karst terrains

by William Z. Savage

Introduction

Subsidence over underground cavities is a common phenomenon, particularly in karst terrains. At the ground surface, subsidence manifests itself by the formation of a depression which may adversely affect adjacent manmade structures. Models of subsidence have been presented by Hackett (1959, 1964), Berry (1960, 1963, 1964a, b), Berry and Sales (1961, 1962) and Salamon (1963, 1964a, b, 1965). These and other subsidence models are reviewed by Voight and Pariseau (1970). In many of these models, as well as in the model introduced here, the subsiding region is assumed to be elastic. This is thought to give a reasonable picture of displacements up to a state of incipient failure.

In what follows, the subsiding region is modeled as an infinitely long elastic layer resting on a rigid base and deforming under its own weight into an opening at its lower edge. An approximate solution for vertical displacements on the ground surface and over the opening is found for the case when the layer thickness is much greater than the width of the opening. This solution, based on the superposition of solutions to two boundary-value problems, differs from previous solutions in that the weight of the subsiding layer is explicitly included. Because of the restricted form of the boundary conditions, this solution is most appropriate for predicting subsidence up to incipient failure in karst terrains. 
The two boundary-value problems

Consider a homogeneous and isotropic elastic layer of thickness $h$, under its own weight, overlying an opening of width $2 a$ (figure 1). The opening is considered to be of great length relative to its width (2a) so that the layer is in a state of plane strain. This general problem can be solved by the superposition of solutions to two separate problems. 


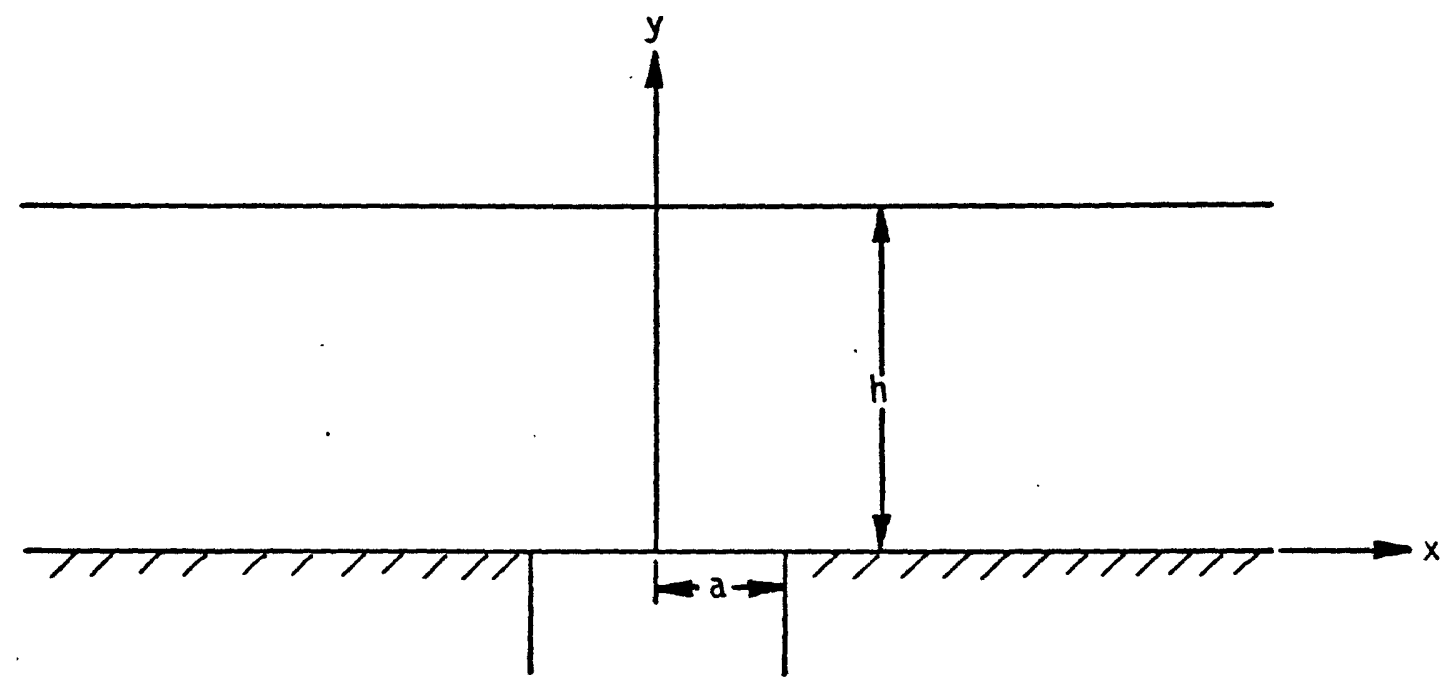

Figure 1.--Elastic layer of thickness $h$ overlying an opening of width $2 a$. 
The first problem is to determine the response of an infinitely wide layer of thickness $h$ to gravity only. The stresses at $y=0$ are

$$
\begin{array}{cc}
\sigma_{x y}(x, 0)=v(x, 0)=0 & x^{2} \geq 0, \text { and } \\
\sigma_{y y}(x, 0)=-o g h & x^{2} \geq 0,
\end{array}
$$

and at $y=h$ are

$$
\sigma_{y y}(x, h)=\sigma_{x y}(x, h)=0 \quad x^{2} \geq 0
$$

A solution for stresses which satisfies equilibrium and compatibility is

$$
\begin{aligned}
& \sigma_{y y}=\rho g(y-h) \\
& \sigma_{x y}=0 \\
& \sigma_{x}=\sigma_{z}=\frac{v}{1-v} \rho g(y-h) .
\end{aligned}
$$

The layer is assumed to be constrained from lateral displacement so

$$
\varepsilon_{x}=\varepsilon_{z}=0, \quad 0 \leqslant x^{2} \leqslant \infty \text { and } 0 \leqslant y \leqslant h
$$

and

$$
\varepsilon_{y}=\frac{(1+v)(1-2 v)}{E(1-v)} \rho g(y-h)
$$

which leads to the vertical displacements, due to gravity only,

$$
v=\frac{(1+v)(1-2 v)}{E(1-v)} \rho g y(y / 2-h)
$$

The second problem is the determination of the response of the layer to an opening of width $2 a$ at its base. Directly over the opening, equilibrium requires that the total vertical stress vanish. Thus on $y=0$,

$$
\begin{aligned}
& \sigma_{y y}(x, 0)=\left\{\begin{array}{cc}
\rho g h & x^{2}<a^{2} \\
0 & x^{2}>a^{2}
\end{array},\right. \\
& \sigma_{x y}(x, 0)=0 \quad x^{2}>0, \text { and } \\
& v(x, 0)=0 \quad x^{2}>a^{2},
\end{aligned}
$$


and on $y=h$

$$
\begin{array}{lll}
\sigma_{x y}(x, h)=0 & x^{2} \geqslant 0 & \text {, and } \\
\sigma_{y y}(x, h)=0 & x^{2} \geqslant 0 &
\end{array}
$$

A solution to this problem for the case $h / a \gg 1$ is given by Sneddon and Lowengrub (1969, p. 62-66).

Vertical displacements at the roof of the opening, that is at $y=0$, as given by Sneddon and Lowengrub (1969), are

$$
v(n, 0)=-v_{0}\left[1-\eta^{2}\right]^{1 / 2}\left[1+\frac{m_{1}}{2 \delta^{2}}+\frac{4 m_{1}^{2}-m_{3}}{16 \delta^{4}}-\frac{\left[1+2 \eta^{2}\right]}{24 \delta^{4}} m_{3}\right]+0\left(\delta^{-6}\right) \quad 0<n<1,
$$

where $n=x / a, v_{0}=\frac{2\left[1-v^{2}\right] p g h a}{E}, \delta=h / a$,

and the notation $0\left(\delta^{-6}\right)$ means that terms of order $(a / h)^{-6}$ and smaller are omitted. Here $m_{1}=2.350$, and $m_{3}=9.962$.

Vertical displacements on the free surface, $y=h$, are given by Sneddon and Lowengrub (1969, eq. 2.11.7, p. 64) as

$$
v(n, \delta)=-2 v_{0} \delta_{\delta}^{\infty} g(\xi)\left[\frac{\sinh \xi \delta+\xi \delta \cosh \xi \delta}{2 \xi \delta+\sinh 2 \xi \delta}\right] \cos \xi \pi d \xi,
$$

where $g(\xi)=\delta^{1} \phi(t) \mathrm{Jo}(\xi \mathrm{t}) \mathrm{dt}$

and

$$
\phi(t)=t\left[1+\frac{m_{1}}{2 \delta^{2}}+\frac{m_{1}^{2}}{4 \delta^{4}}-\frac{\left(2 t^{2}+1\right) m_{3}}{16 \delta^{4}}\right]+0\left(\delta^{-6}\right) \quad .
$$

Substitution of equation 7 in equation 6 and integrating leads to

$$
g(\xi)=\left[1+\frac{m_{1}}{2 \delta^{2}}+\frac{m_{1}^{2}}{4 \delta^{4}}-\frac{3 m_{3}}{16 \delta^{4}}\right] \frac{1}{\xi} J_{1}(\xi)+\frac{m_{3}}{4 \delta^{4}} \frac{J_{2}(\xi)}{\xi^{2}}+0\left(\delta^{-6}\right),
$$


and thus,

$$
\begin{aligned}
& v(n, \delta)=-2 v_{0}\left[\left[1+\frac{m_{1}}{2 \delta^{2}}+\frac{m_{1}^{2}}{4 \delta^{4}}-\frac{3 m_{3}}{16 \delta^{4}}\right] \delta_{\delta}^{\infty}\left[\frac{\sinh \xi \delta+\xi \delta \cosh \xi \delta}{2 \xi \delta+\sinh 2 \xi \delta}\right] J_{1}(\xi) \cos \xi n \frac{d \xi}{\xi}\right. \\
& \left.+\frac{m_{3}}{4 \delta^{4}} \delta_{\delta}^{\infty}\left[\frac{\sinh \xi \delta+\xi \delta \cosh \xi \delta}{2 \xi \delta+\sinh 2 \xi \delta}\right] J_{2}(\xi) \cos \xi n \frac{d \xi}{\xi^{2}}\right]+0\left(\delta^{-6}\right) .
\end{aligned}
$$

Evaluation of vertical displacements on the free surface

The integrals in equation 9 can be found approximately by using filon's quadrature (Sneddon, 1951, p. 519). Using this procedure, when $\delta \gg>1$, say 10 or greater, sufficient accuracy is obtained by excluding terms of order $\delta^{-4}$ and smaller. Vertical displacements of the free surface are then given by the approximate expression,

$$
v(n, \delta)=-2 v_{0}\left[1+\frac{m_{1}}{2 \delta^{2}}\right] \int_{0}^{\infty} M(\xi \delta) J_{1}(\xi) \cos \xi \frac{d \varepsilon}{\xi},
$$

where $M(\xi \delta)=\frac{\sinh \xi \delta+\xi \delta \cosh \xi \delta}{2 \xi \delta+\sinh 2 \xi \delta}$

An alternative to quadrature is to follow sneddon (1951) and replace $M(\xi \delta)$ in equation 10 by the approximate expression,

$$
M(\xi \delta) \approx M_{1}(\xi \delta)=(1+\xi \delta) e^{-\xi \delta}-1 / 2[1+2 \xi \delta] e^{-\alpha \xi \delta} \quad \text {. }
$$

This approximation is best at large values of $\xi \delta$ where $M(\xi \delta)=M_{1}(\xi \delta)=$ $(1+\xi \delta) e^{-\xi \delta}$, provided $\alpha>1$. For smaller values of $\xi \delta$, the parameter $\alpha$ is chosen in such a way that the area under the curve $M_{1}(\xi \delta)-M(\xi \delta)$ is as small as possible. This condition is best satisfied when $\alpha=1.85$. 
Substituting equation 11 in the integral $\delta_{\delta}^{\infty} M(\xi \delta) J_{1}(\xi) \cos \xi \pi \frac{d \xi}{\xi}$ gives the approximation, \& $M_{1}(\xi \delta) J_{1}(\xi) \cos \xi n \frac{d \xi}{\xi}=I_{1}+\delta I_{2}-1 / 2 I_{3}-\delta I_{4}$,

where

$$
\begin{aligned}
& I_{1}=\delta \mathrm{e}^{-\delta \xi} J_{1}(\xi) \cos \xi \frac{d \xi}{\xi}, \\
& I_{2}=\infty \mathrm{e}^{-\delta \xi} J_{1}(\xi) \cos \xi n d \xi, \\
& I_{3}=\infty \mathrm{e}^{-\alpha \delta \xi} J_{1}(\xi) \cos \xi \frac{d \xi}{\xi}, \text { and } \\
& I_{4}=\delta \mathrm{e}^{-\alpha \delta \xi} J_{2}(\xi) \cos \xi n d \xi,
\end{aligned}
$$

The integrals $I_{1}, I_{2}, I_{3}, I_{4}$, are found by methods described in Sneddon (1951). The results are

$$
\begin{aligned}
& I_{1}=\frac{1}{\sqrt{2}}\left[R_{1}^{2}+1+\delta^{2}-n^{2}\right]^{1 / 2}-\delta, \\
& I_{2}=1-\frac{1}{\sqrt{2} R_{1}^{2}}\left[\delta\left[R_{1}^{2}+1+\delta^{2}-n^{2}\right]^{1 / 2}+n\left[R_{1}^{2}-\left[1+\delta^{2}-n^{2}\right]\right]^{1 / 2}\right], \\
& I_{3}=\frac{1}{\sqrt{2}}\left[R_{2}^{2}+1+\alpha^{2} \delta^{2}-n^{2}\right]^{1 / 2}-\alpha \delta, \text {, and } \\
& I_{4}=1-\frac{1}{\sqrt{2} R_{2}^{2}}\left[\delta\left[R_{2}^{2}+1+\alpha^{2} \delta^{2}-n^{2}\right]^{1 / 2}+n\left[R_{2}^{2}-\left[1+\alpha^{2} \delta^{2}-n^{2}\right]\right]^{1 / 2}\right],
\end{aligned}
$$

where $R_{1}^{2}=\left[\left[1+\delta^{2}-n^{2}\right]^{2}+4 \delta^{2} \eta^{2}\right]^{1 / 2}$ and $R_{2}^{2}=\left[\left[1+\alpha^{2} \delta^{2}-n^{2}\right]^{2}+4 \alpha^{2} \delta^{2} n^{2}\right]^{1 / 2}$. 
Vertical displacements on the free surface and

over the opening

Displacements due to the weight of the layer deforming into the opening are now obtained by superposition of the displacements obtained in problems 1 and 2. For $y=0$, this yields the vertical displacements

$$
v(n, 0)=-\frac{2\left[1-v^{2}\right]_{\rho} g h a}{E}\left[1+\frac{m_{1}}{2 \delta^{2}}\right]\left[1-n^{2}\right]^{1 / 2},
$$

and for $y=h$, the vertical displacements

$$
v(n, h)=-\frac{(1+v)(1-2 v)}{2 E(1-v)} \rho g h^{2}-\frac{4\left[1-v^{2}\right] \rho g h a}{E}\left[1+\frac{m_{1}}{2 \delta^{2}}\right]\left[I_{1}+\delta I_{2}-1 / 2 I_{3}-\delta I_{4}\right] .
$$

We can now write equation 12 in the dimensionless form

$$
\frac{v(n, 0)}{v_{0}\left[1+\frac{m_{1}}{2 \delta^{2}}\right]}=-\left[1+n^{2}\right]^{1 / 2} \text {. }
$$

Since the first term on the right in equation 13 is a constant,

$$
\begin{aligned}
& v_{g}=\frac{(1+v)(1-2 v) \rho g h^{2}}{2 E(1-v)} \text {, we have } \\
& \frac{v(n, \delta)+v_{g}}{m_{1}}=-\left[I_{1}+\delta I_{2}-1 / 2 I_{3}-\delta I_{1}\right]
\end{aligned}
$$

for the vertical displacements on the free surface.

Vertical displacements on $y=h$ for $\delta=10$ predicted by Filon's quadrature of equation 10 and by equation 15 are the same to 3 decimal places. Vertical displacements on $y=h$, predicted by equation 15 for three values of the ratio $\delta=h / a$, are shown in figure 2 . Vertical displacements over the hole (on $y=0$ ) predicted by equation 14 are shown in figure 3 . 


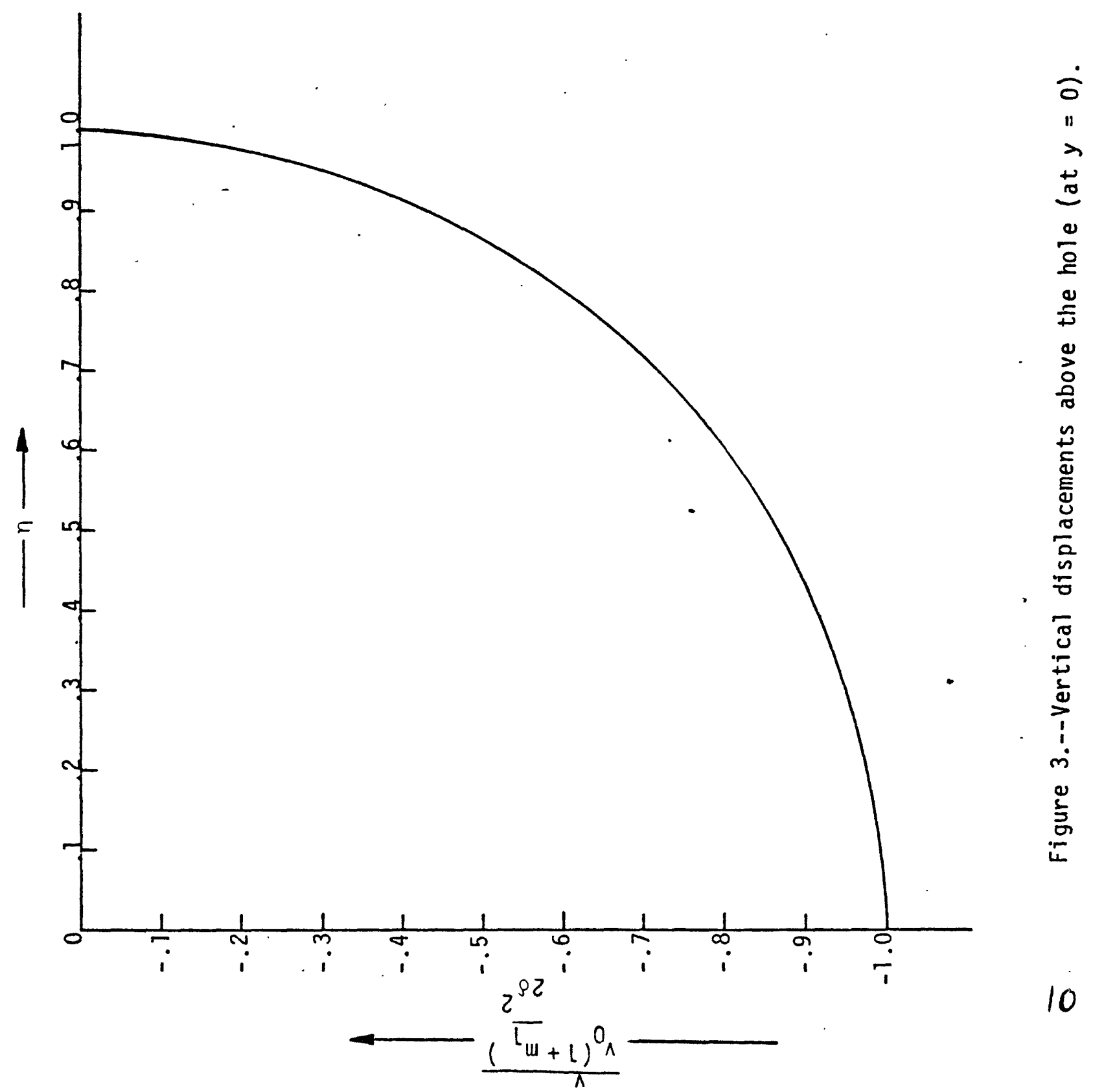




\section{Discussion of resul.ting displacements}

Vertical displacements on the free surface, shown in figure 2, are seen to decrease as $h$, the layer thickness, increases relative to the width of the opening, 2a. However, a broader trough of subsidence is formed as $\delta=h / a$ increases. Additionally, because $v_{0}=\frac{2\left(1-v^{2}\right) \rho g h a}{E}$ and $v_{g}=\frac{(1+v)(1-2 v)}{2 E(1-v)} \rho g h^{2}$, increasing Young's modulus, $E$, causes a decrease in free-surface vertical displacements. Conversely, increasing the density, $\rho$, and $h$ or a causes an increase in vertical displacements at the surface. Also, increasing Poisson's ratio, $v$, causes a decrease in $v_{0}$ and $v_{g}$ and hence $a$ decrease in vertical displacements at the ground surface. Note that the width of the subsidence trough is controlled only by the ratio $h / a$ and is not affected by changing $v_{0}$ or $v_{g}$. Of equal interest is the formation of a ridge at the edge of the subsidence trough. This phenomenon is clearest in the case $\delta=h / a=10$ (fig. 2). Vertical displacements over the hole, as shown in figure 3 , will be increased as $\rho, h$, or a is increased and will be decreased as $\delta, E$, or $v$ is increased.

Displacements over the hole, at $y=0$, approximate actual field conditions as displacements for $x^{2}>a^{2}$ will not vanish unless the subsiding layer overlies a very stiff base. This is also true for shear stresses at $y=$ 0 when $x^{2}>a^{2}$. The assumption that these stresses vanish implies that the layer is free to slide towards the hole as it subsides. Again this would be approximately true in the case of a relatively soft layer overlying a very stiff layer. Recognizing these restrictions, the present solution is best used as a model of subsidence in a karst terrain where a thick and relatively soft soil layer overlies solution cavities in much stiffer limestone or dolomite. 


\section{References}

Berry, D. S., 1960, An elastic treatment of ground movement due to mining, Part I: Journal of Mechanics and Physics of Solids, v. 8, no. 4, p. 280-292.

1963, Ground movement considered as an elastic phenomenon: Mining Engineer, no. 37 , p. 28-39.

1964a, A theoretical elastic model of the complete region affected by mining a thin seam: Sixth Symposium on Rock Mechanics, Rolla, 1964, Proceedings, p. 310-329. 1964b, The ground considered as a transversely isotropic material: International Journal of Rock Mechanics and Mining Sciences, v. I, no. 2, p. $159-167$.

Berry, D. S., and Sales, T. W., 1961, Elastic treatment of ground movement due to mining, Part II: Journal of Mechanics and Physics of Solids, v. 9, no. 1 , p. 52-62. 1962, An elastic treatment of ground movement due to mining, Part III: Journal of Mechanics and Physics of Solids,. v. 10, no. 2, p. 73-83. Hackett, P., 1959, An elastic analysis of rock movement caused by mining:

Institute of Mining Engineers Transactions, v. 118, pt. 7, p. 421-433. 1964, Prediction of rock movement by elastic theory compared with insitu measurement: Rock Mechanics and Engineering Geology, Supplement 1, p. $88-102$.

Salamon, M. D. G., 1963, Elastic analysis of displacements and stresses induced by the mining of seam or reef deposits, Part I: Journal of South African Institute of Mining and Metallurgy, v. 64, p. 128-149. 
Salamon, M. D. G., 1964a, Elastic analysis of displacements and stresses induced by the mining of seam or reef deposits, Part II: Journal of South African Institute of Mining and Metallurgy, v. 64, p. 197-218. 1964b, Elastic analysis of displacements and stresses induced by the mining of seam or reef deposits, Part III: Journal of South African Institute of Mining and Metallurgy, v. 64, p. 468-500. 1965. Elastic analysis of displacements and stresses induced by the mining of seam or reef deposits, Part IV: Journal of South African Institute of Mining and Metallurgy, v. 65, p. 319-338. Sneddon, I. N., 1951, Fournier transforms: New York, McGraw-Hi11, 542 p. Sneddon, I. N., and Lowengrub, M., 1969, Crack problems in the classical theory of elasticity: New York, John Wiley, 221 p. Voight, B., and Pariseau,.W., 1970, State of predictive art in subsidence engineering: American Society of Civil Engineers, Proceedings, Journal of Soil Mechanics and Foundations Division, v. 96, SM2, p. 721-750. 